\title{
Editorial
}

\section{Bioequivalence studies of reformulated pharmaceuticals newly off patent}

Studies aiming to show that two drugs are bioequivalent in their actions appear occasionally in peer review journals. They are usually regarding a drug that has recently gone off patent and the new product is tested against a well-established original version. The new products are either entirely produced by a new manufacturer and already licensed by a responsible authority, or are compounded from imported bulk purchased from another manufacturer. There are now pharmaceutical factories in several countries who specialize in manufacturing such bulks for firms that will compound them locally into formulations with a new proprietary name. Lowering the cost and increasing demand is what drives this enterprise. This is one mechanism that can make the newly compounded drug more affordable for patients. However, it can also produce a less effective and even unsafe formulation if the end product has not been carefully compounded and properly tested to establish equivalence and safety. It is ultimately the bulk product that determines equivalence of action and it should be independently evaluated before marketing.

It is important to know is that published studies of equivalence are not a legal requirement in Thailand or most other countries in this region. A drug compounded from bulk products must have the bulk manufacturer approved by the country of the bulk's origin. The bulk compounding should be conducted by a firm that is approved by the National Registration Authority (NRA) in the compounder's country. The compounding process must be conducted according to current internationally acceptable, for example World Health Organization, Good Manufacturing Practice (GMP) guidelines. All data pertaining to this must be in the open realm. Subsequent bulk materials purchased from a different origin must again be approved by the original bulk manufacturer's market NRA and again that of the compounding country that will mark the product. This is embedded in clear

Correspondence to: Editorial Office of Asian Biomedicine, Faculty of Medicine, Chulalongkorn University, Bangkok 10330, Thailand.E-mail: chulamed@md.chula.ac.th guidelines [1-3]. One must hope that these guidelines are being fully complied with, and may not be the case in some countries. These studies usually take place with support from the bulk maker or compounding manufacturer. A quick look at the Thai pharmaceutical list shows that making duplicates of reborn patent free products with bulk imported from China, India, Brazil and other courtiers is a growth industry. It is also one that can benefit us all when done correctly. Reading such a bioequivalence study in a peer review journal can be reassuring to doctors and patients who might benefit from reduced prices. Nevertheless, many would consider such publications as a form of advertising; something that editors avoid in peer review journals. However, one might also argue that such studies, if performed properly, honestly, and in accordance with international guidelines, are likely to support formulations that are more affordable, and thus beneficial to global health. Furthermore, patented drugs originating in industrial countries are only licensed after extensive phase I-III trials. In most cases, this is later followed by a large post-marketing study that should detect rare late adverse side effects and other problems. This is not usually done with drugs compounded from bulk. Thus, a properly performed bioequivalence study can offer some reassurance to end users that is currently not generally available elsewhere. We have therefore decided to publish the paper from Jullangkoon et al. [4] even though it did receive support from the compounding manufacturer. The bulk product from Brazil had been previously accepted by the Brazilian and Thai NRAs and the study by Jullankoon et al. was performed and reported in an internationally acceptable manner. Thus, even though this is a commercially motivated study, it documents equivalence and lack of adverse side effects. We will accept other selected bioequivalence studies that are pertinent to the southeast Asian region. They should state the origin of the bulk and that the manufacturer is approved by the NRA, and the compounding conforms to current GMP guidelines. Any potential conflict of interest must be declared, and the clinical study conducted in accordance with international ethics guidelines. 


\section{References}

1. Chen, ML, Shah V, Patnaik R, Adams W, Hussain A, Conner D, et al. Bioavailability and bioequivalence: an FDA regulatory overview. Pharm Res. 2001; 18: 1645-50.

2. Raw AS, Furness MS, Gill DS, Adams RC, Holcombe FO, Yu LX. Regulatory considerations of pharmaceutical solid polymorphism in Abbreviated New Drug Applications (ANDAs). Adv Drug Deliv
Rev. 2004; 56:397-414.

3. WHO Tech Rep Ser. Additional guidelines for organizations performing in vivo bioequivalence studies. Geneva 2006.

4. Jullangkoon, M, Jarurathanasirikul S, Ainlang N. Bioequivalence study of cefepime intramuscular injection in healthy volunteers. Asian Biomed. 2014; 8: 237-40 